Revue des patrimoines

\title{
« De la demande du lecteur à la reconstitution d'un fonds : les archives du Haras national de Villeneuve-sur-Lot »
}

Pascal De Toffoli

\section{OpenEdition}

Journals

Édition électronique

URL : http://journals.openedition.org/insitu/9745

DOI : 10.4000/insitu.9745

ISSN : 1630-7305

Éditeur

Ministère de la culture

Référence électronique

Pascal De Toffoli, « «De la demande du lecteur à la reconstitution d'un fonds : les archives du Haras national de Villeneuve-sur-Lot » », In Situ [En ligne], 18 | 2012, mis en ligne le 31 juillet 2012, consulté le 22 avril 2019. URL : http://journals.openedition.org/insitu/9745 ; DOI : 10.4000/insitu.9745

Ce document a été généré automatiquement le 22 avril 2019

\section{(c) $(1) \odot$}

In Situ Revues des patrimoines est mis à disposition selon les termes de la licence Creative Commons Attribution - Pas d'Utilisation Commerciale - Pas de Modification 4.0 International. 


\title{
« De la demande du lecteur à la reconstitution d'un fonds : les archives du Haras national de Villeneuve-sur- Lot»
}

\author{
Pascal De Toffoli
}

1 Les Archives départementales de Lot-et-Garonne ont mené entre 2005 et 2010 une opération de préservation d'un ensemble de carnets à souches de saillies du dépôt d'étalons de Villeneuve-sur-Lot conduisant à reconstituer le patrimoine écrit de cet établissement.

2 Comment l'intérêt d'un chercheur, spécialiste de l'histoire du cheval, pour une collection «oubliée » de carnets de saillies, versée il y a 26 ans de cela, a-t-il su convaincre les Archives départementales de s'intéresser et de conserver ces documents sériels, encombrants et difficilement exploitables en l'état, pour dès lors s'attacher à reconstituer le fonds du dépôt de Villeneuve-sur-Lot?

3 L'intérêt pour ces carnets à souches de saillies s'est progressivement révélé pour dépasser la seule action menée depuis leur versement en 1978, à savoir un simple stockage, et a fait naître le besoin et l'envie de rassembler le fonds du dépôt d'étalons. Avant de statuer sur le sort de ces carnets, il s'agissait d'apprécier leur valeur, leur utilité et savoir si ces documents se suffisaient à eux-mêmes. Cela a fait naître un questionnement autour de l'évaluation à pratiquer et autour des critères de tri pour que cette série organique conserve tout son sens pour la généalogie équine. Nous, archivistes, étions ainsi confrontés à des documents sériels déconnectés de leur origine que nous devions appréhender et faire fructifier pour les rattacher aux documents émanant plus largement du dépôt d'étalons.

4 L'objectif du service était de proposer un ensemble cohérent non seulement pour la généalogie équine mais également nécessaire à l'étude de la structure même dans son fonctionnement et ses lieux. La solution semblait passer par deux opérations 
intrinsèquement liées: la conservation et le classement des carnets à souches et la collecte des archives encore conservées par le dépôt d'étalons.

Après une brève présentation de ce lieu dédié au cheval, seront abordées non seulement les raisons qui ont conduit à s'intéresser aux carnets à souches de saillies, dans leur évaluation et leur traitement archivistique, mais aussi à la collecte et au contenu du reste $\mathrm{du}$ fonds d'archives. Nous pourrons alors nous demander si les documents de nouveau rassemblés étaient bien complémentaires, justifiant notre implication et répondant d'autant aux besoins documentaires à la fois du haras qu'aux sujets de recherche des lecteurs et étudiants.

\section{Présentation du dépôt d'étalons de Villeneuve-sur-Lot}

\section{Histoire}

6 À la suite du décret impérial du 4 juillet 1806 rétablissant les Haras nationaux, un des trente dépôts d'étalons est ouvert provisoirement, à l'automne 1807, au chef-lieu du département de Lot-et-Garonne, au cœur de la ville d'Agen, dans l'hôtel de Loss ${ }^{1}$. Puis, il est transféré en 1810 à Villeneuve-sur-Lot, à l'emplacement de l'ancien couvent des Cordeliers, où une réduction budgétaire le contraint à fermer en 1832. Si cette première période d'activité demeure très largement méconnue du fait des sources locales inexistantes, il semble que le dépôt de Libourne ait prit la relève jusqu'à ce que l'ordonnance royale du 3 juin 1842 ne le rétablisse, pour un fonctionnement effectif en 1845 dans les mêmes locaux².

\section{Ressort}

7 Sa circonscription, d'abord limitée aux départements de Lot-et-Garonne et Tarn-etGaronne avec leurs dix stations de monte, est étendue au département du Lot en 1850. À l'exception de quelques stations transférées et fermées, le ressort ne changera plus au $\mathrm{XIX}^{\mathrm{e}}$ siècle et pendant une bonne partie du $\mathrm{XX}^{\mathrm{e}}$ siècle. La circonscription comptera alors, en dehors du dépôt de Villeneuve, jusqu'à dix-neuf stations de monte. Puis elle est profondément modifiée à deux reprises : en 1971, où elle perd, au profit de Rodez, les départements du Lot et de Tarn-et-Garonne, pour englober le département de la Gironde ; et en 1996, où le département de la Dordogne vient la compléter.

8 Aujourd'hui le dépôt, en dehors de son centre technique, ne comporte plus que deux stations de monte, Bazas et Villeréal, appelées à fermer respectivement en 2012 et 2013.

\section{Situation géographique}

9 Situé au cœur de la bastide du XIII ${ }^{e}$ siècle (fig. $\left.\mathbf{n}^{\circ} \mathbf{1}\right)$ (fig. $\left.\mathbf{n}^{\circ} \mathbf{2}\right)$, ce site intra-muros s'étend sur une surface de 1,7 hectare et dispose de 7 hectares de prairies bordant la ville. Le dépôt figure dans une aire de mise en valeur de l'architecture et du patrimoine, du fait de la proximité de l'église Saint-Étienne. Les bâtiments, édifiés pour la plupart entre 1876 et 1910, s'organisent autour d'un parc arboré comportant en son centre l'ancienne maison de direction et le manège, et, en périphérie, les bureaux, la forge, la sellerie, les deux écuries et la remise. 


\section{Figure 1}

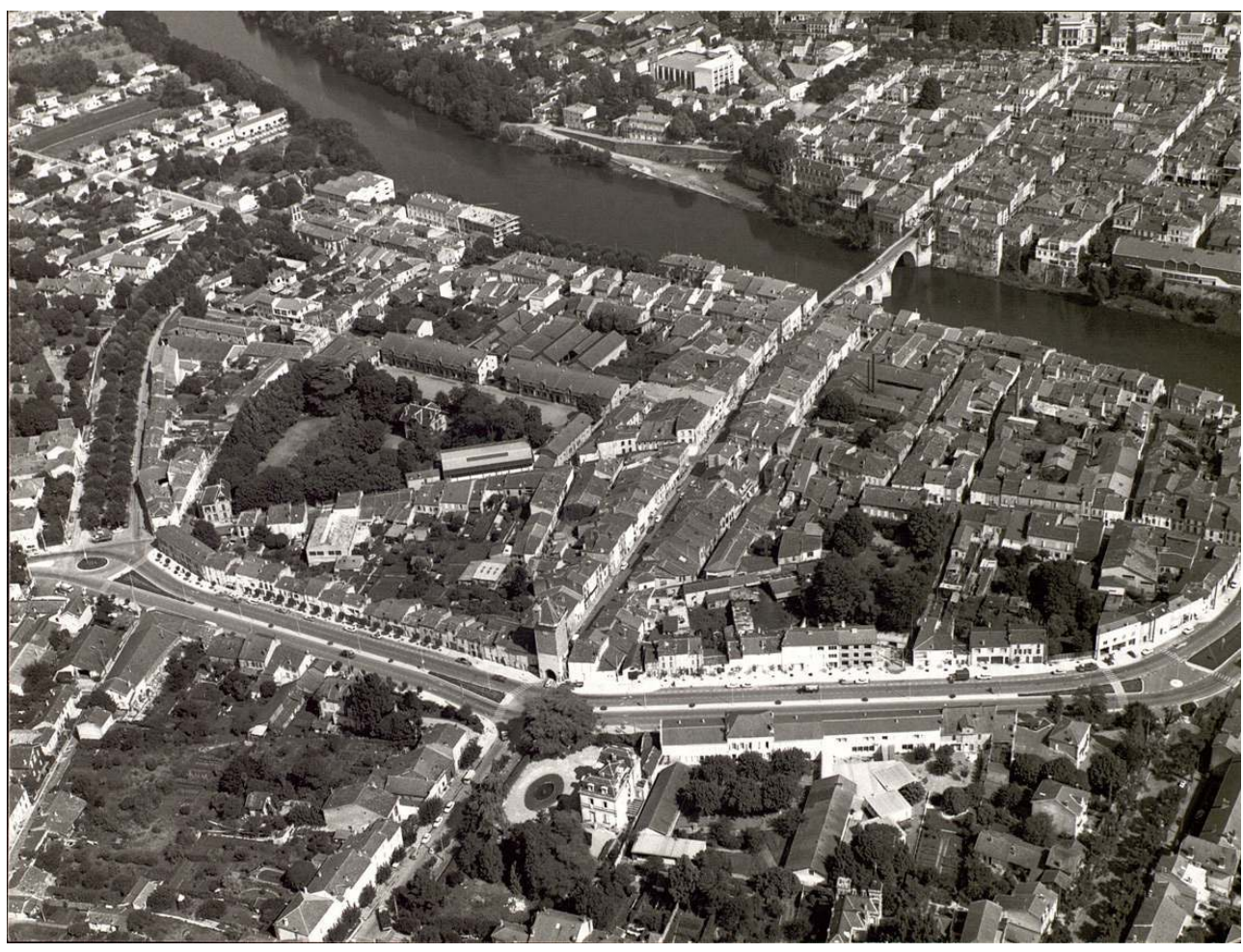

Le dépôt d'étalons de Villeneuve-sur-Lot vu du ciel, vers 1960. Phot. Delvert, Ray. Document conservé aux Archives municipales de Villeneuve-sur-Lot sous la cote 3 Fi 26.

Repro. Vigué, Marie. @ Conseil général de Lot-et-Garonne, service des Archives départementales, 2011. 
Figure 2

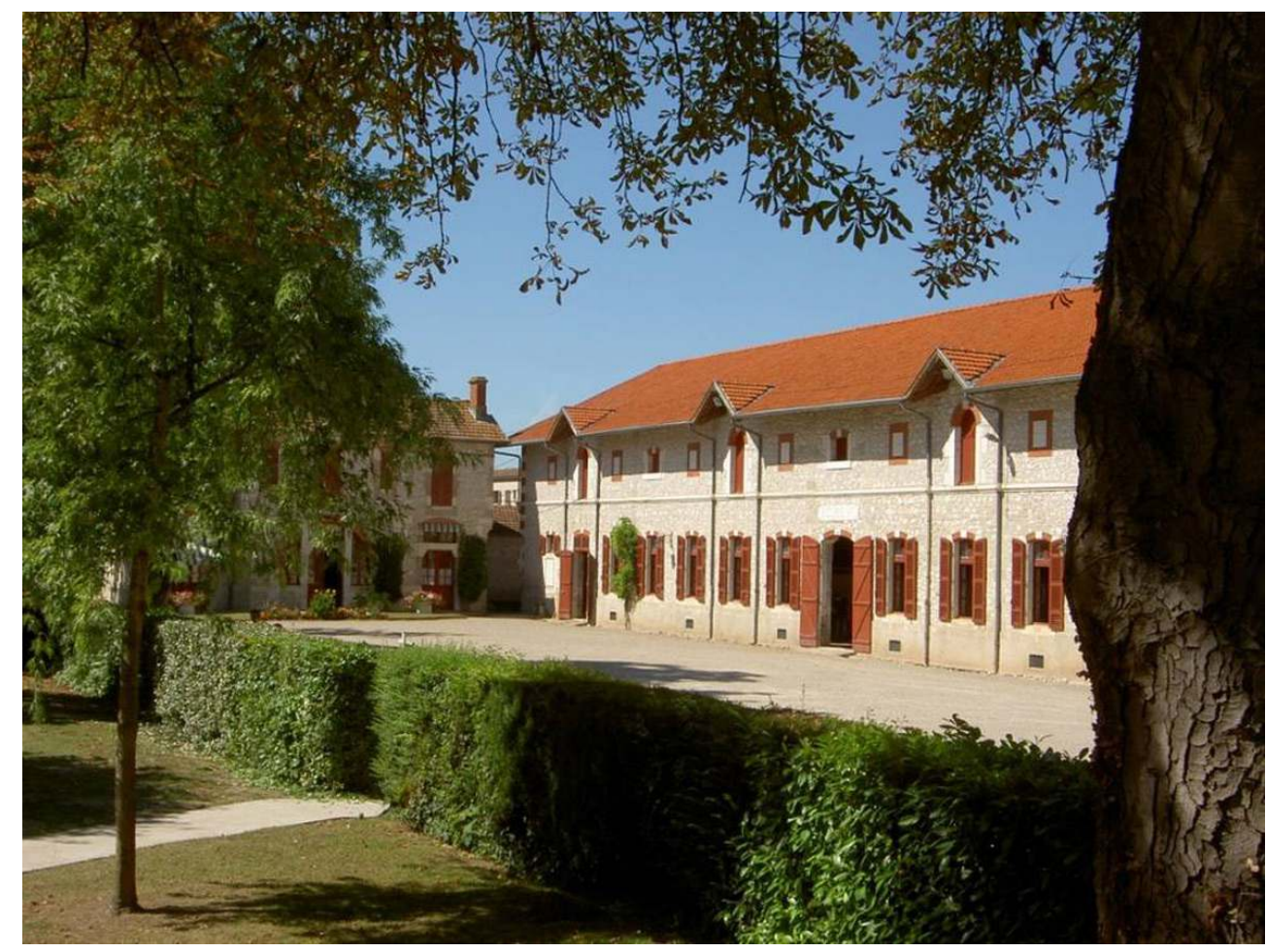

Les écuries du dépôt d'étalons de Villeneuve-sur-Lot.

Phot. Niquin, V., des Haras nationaux. (c) Institut français du cheval et de l'équitation, [2000].

\section{Activité}

Le dépôt d'étalons de Villeneuve gère actuellement 21 étalons : 11 animaux sont présents physiquement sur ses sites et 10 animaux de trait sont en location chez des éleveurs. Sa production annuelle, qui atteignait 2525 saillies [avec 43 étalons] en 1851, se borne aujourd'hui à environ 80 juments saillies.

\section{Comment ce patrimoine nous est-il venu en « conscience »?}

11 Les archivistes possèdent tous dans leurs magasins des fonds non inventoriés qui sommeillent compte tenu de leur nombre en attente de traitement. Jusque-là, ces documents se signalaient d'eux-mêmes, par leur présence massive et brute, dans de grands cartons disparates stockés dans un magasin du dernier étage. Cette relégation spatiale se doublait d'un désintérêt de notre part pour cet ensemble documentaire rencontré à l'occasion de déplacements pour gérer l'espace, les collections et prévoir leur entretien.

Intrigués par le contenu de ces cartons, nous en sommes venus à extraire furtivement un carnet à souches de saillies pour y jeter un œil amusé et dénué de toute ambition archivistique. Cet ensemble était aussitôt oublié, étant donné que l'espace de stockage 
était suffisant pour repousser sine die cette question et que les documents, d'une valeur scientifique inconnue, n'avaient jamais fait l'objet d'une demande de communication.

Cette documentation est demeurée enfouie jusqu'à la réception d'un courrier du 18 juillet 2005 de Guy Bideault, ancien directeur du dépôt d'étalons de Saint-Lô, spécialiste de la généalogie équine et chercheur sur l'histoire des chevaux de selle. Guy Bideault souhaitait consulter des carnets de saillies de plusieurs étalons et avait été orienté vers notre service par l'ancien directeur du dépôt d'étalons de Villeneuve, M. Valentin, qui les avait versés aux Archives départementales en 1978. Nous avons alors véritablement pris conscience que ces documents pouvaient être communiqués au regard de leur intérêt historique pour le Stud-book. En outre, une telle recherche exigeait l'identification et le regroupement des carnets concernant les étalons étudiés, le bordereau de versement existant - réduit à une page - n'étant d'aucun secours. Avec l'aide de Danièle Roulet, chargée de l'entretien des collections, nous avons, au prix d'un long travail de recherche et de dépoussiérage, donné en communication ces quelques documents pour la plus grande satisfaction de Guy Bideault.

\section{Remise en cause de nos pratiques de collecte et de traitement des documents}

14 Cette demande nous a interpellés sur notre pratique de collecte et de traitement de fonds qui contrariait nos missions de communication et de valorisation. Comment appréhender des documents gelés du fait de notre méconnaissance de leur intérêt? Et combien de temps encore pouvions-nous conserver ces documents, inexploitables en l'état, sans une mise à disposition des lecteurs ?

Si la collecte demeure bien souvent une opportunité et n'est pas systématiquement suivie du classement des documents les services d'archives s'efforcent de réduire les arriérés de traitement des fonds en établissant des programmes de classements prioritaires basés sur la valeur des documents et sur les besoins des lecteurs. Ainsi, dès l'instant où un lecteur s'intéressait à ce fonds, notre service se devait de répondre à la demande et pouvait difficilement s'abriter derrière la règle "non classé = non communicable » sous peine d'entraver la recherche historique.

Cela a fait naître des questions pratiques relatives à la gestion des rares, mais réelles, demandes de communication portant sur ces documents sales, stockés de façon désordonnée et non référencés. Ne rien faire équivalait, pour toute nouvelle demande d'un chercheur, à rencontrer les mêmes difficultés et à y consacrer un temps considérable de dépoussiérage et de mise en ordre.

Il fallait donc pouvoir proposer aux lecteurs des conditions normales de consultation et de recherche reposant sur le repérage des documents préalablement nettoyés. Par ailleurs, le stockage même, dans des grands cartons ouverts à la poussière, participait à une mauvaise conservation des documents, faute de dépoussiérage régulier et de conditionnement approprié. Aussi, avant d'entreprendre une opération coûteuse de mise en ordre, d'inventoriage et de mise en boîte, il convenait de nous assurer que ce matériau documentaire méritait d'être conservé. 


\section{Quand vient le temps de statuer sur le sort des carnets à souches de saillies} ces documents. Cette opération était rendue difficile par la déconnexion de cet ensemble sériel des compléments susceptibles d'être conservés par le dépôt d'étalons, et par l'absence d'instruction de tri - la circulaire de la direction des archives de France sur le sujet n'ayant été publiée qu'en janvier 2007.

19 Jusque-là, la première réflexion du service sur le sort de ces carnets remontait à 1981, date à laquelle ces documents avaient échappé à une élimination partielle proposée par le directeur des Archives départementales. À la conservation d'une année témoin, l'année 3 tous les 20 ans $(1853,1873,1893,1913,1933,1953$ et 1960), le chef de service des Haras et de l'équitation avait répondu le 29 décembre 1981 en ces termes: "Je ne peux pas accepter une telle proposition: la destruction des souches de cartes de saillies nous priverait de toute possibilité d'être renseigné, avec précision, sur l'élevage de cette région ». L'archiviste avait obtempéré et replongé ces documents dans un long sommeil.

L'expertise contemporaine devait nécessairement croiser les avis des deux réseaux, celui des Haras nationaux et celui de la Direction des Archives de France, tout en observant les arguments du chercheur. Guy Bideault a de ce point de vue suscité et stimulé la réflexion en sensibilisant les Archives au contenu des documents. Il était pour sa part favorable à une conservation intégrale des carnets pour l'histoire du cheval et, en particulier, parce qu'ils étaient les seuls à répertorier une demi-race de sang arabe. Après être intervenu personnellement auprès de Monique Le Bon, responsable du pôle hippique du dépôt d'étalons de Villeneuve, en ce sens, il suggérait trois solutions : conserver l'intégralité aux Archives départementales en leur adjoignant les registres matricules du haras, les proposer au Haras national de Pompadour, qui gère le fichier de l'ensemble des étalons des Haras nationaux, ou les confier, sous réserve de place disponible, au dépôt d'étalons de Tarbes qui conservait ses propres archives.

21 La position des Haras nationaux, en la personne de Diane de Sainte-Foy, directrice de la communication, consistait à préserver ces documents en les restituant au dépôt de Villeneuve, après que Monique Le Bon soit venue se rendre compte du volume de cet ensemble documentaire. Parallèlement, Monique Le Bon semblait vouloir a contrario nous remettre les autres documents historiques du haras.

Après ces premières réflexions, les Archives de France devaient nous fournir des conseils à l'appui des expériences territoriales. D'abord, Martine Salmon-Dalas, directrice des Archives de Lot-et-Garonne, ne pouvait, au regard de l'espace occupé dans les magasins et du coût de conservation, défendre une conservation intégrale de ces documents d'une grande technicité qui intéressaient seulement «trois chercheurs en France ». Aussi, la tentation d'éliminer ces carnets à souches était grande, compte tenu que cette typologie documentaire n'apparaissait pas dans les inventaires publiés des fonds d'archives des Haras conservés dans d'autres départements. Pour autant, nous ignorions si ces documents étaient encore conservés par les Haras nationaux ou collectés en l'état par les services d'archives, ou s'ils avaient été éliminés avec ou sans l'accord des Archives départementales. Cependant, Mme Salmon-Dalas était intéressée à la conservation des 
carnets relatifs aux étalons de sang arabe, carnets qui ne semblaient pas conservés dans les autres services d'archives.

Au premier abord, l'expertise s'orientait vers un tri sélectif pour ne conserver que des années témoins et la restitution au dépôt de Villeneuve des années non retenues. Heureusement, l'éclairage de Jean-François Le Nail, alors directeur des Archives départementales des Hautes-Pyrénées, qui avait travaillé avec le dépôt d'étalons de Tarbes, fut déterminant. Il écartait d'emblée la possibilité de les confier au dépôt d'étalons de Tarbes par manque de place. En outre, il pointait le caractère épineux du tri qui, s'il reposait sur la conservation des années 0 et 5 et les années de guerre, était révélateur de l'histoire humaine mais s'avérait impropre et incertain appliqué au cheval. $\mathrm{Au}$ final, il conseillait vivement de conserver intégralement ces documents à l'exemple des Pyrénées-Atlantiques.

À l'appui des avis de Guy Bideault et de Jean-François Le Nail, Martine Salmon-Dalas a décidé de préserver l'intégralité de la collection. Elle a, en effet, admis que l'échantillonnage empêcherait toute généalogie équine faute de pouvoir reconstituer la production détaillée, ne serait-ce que d'un étalon. Mais le fait de conserver des années décousues présentait-il un intérêt?

Mais la conservation intégrale était toutefois subordonnée à la reconstitution du fonds par la collecte des documents non versés avec pour objectif de constituer un ensemble homogène et riche pour le public. Ainsi, les sources complémentaires - préfectorales notamment - ne devaient plus constituer la documentation de référence, mais le public devait pouvoir disposer au contraire d'un ensemble riche et homogène de documents produits directement par l'établissement tels que les registres de la monte et les registres matricules des étalons.

\section{La collecte et le traitement des archives}

L'opération a commencé par la collecte des archives du dépôt. Il fallait s'assurer de disposer de l'ensemble du fonds avant de lancer le traitement des carnets à souches de saillies.

En septembre-octobre 2005, après accord des parties, la cellule des archives contemporaines a mené une opération d'identification et de tri des archives historiques du haras, suivie de leur transfert aux Archives départementales. Seul un lot de plans des bâtiments destinés à identifier le propriétaire du dépôt, l'État ou la commune, a été conservé par l'administration des Haras.

Aussitôt entrés, les 3,10 mètres linéaires de documents ont été dépoussiérés, classés, inventoriés et conditionnés. Dans un second temps, en 2009-2010, Samira Méziane, adjoint administratif, a procédé au classement et au conditionnement de la série de carnets à souches de saillies (fig. $\mathbf{n}^{\circ} \mathbf{3}$ ). Ce fonds, coté en sous-série 3 ETP, a été doté d'un répertoire numérique détaillé de 116 pages, en ligne sur notre site Internet ${ }^{3}$. 

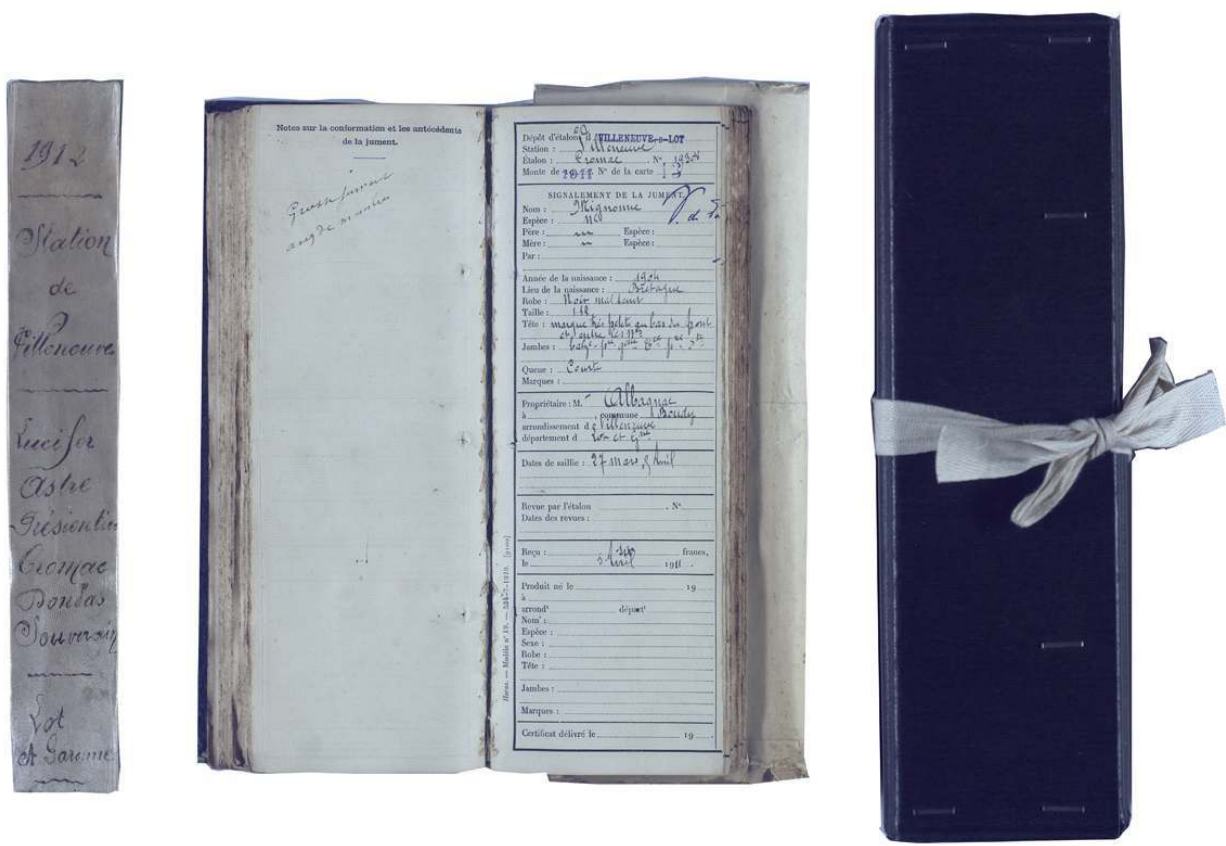

Carnets à souches de saillies de la station de monte de Villeneuve-sur-Lot, et leur boîte de conservation, 1911-1912. Documents conservés aux Archives départementales de Lot-et-Garonne sous les cotes 3 ETP 845-846.

Repro. Vigué, Marie. (c) Conseil général de Lot-et-Garonne, service des Archives départementales, 2011.

Cette opération, mobilisant une personne pendant environ 85 jours, a non seulement permis de gagner de la place dans le dépôt des archives, mais a, en outre, reconstitué un fonds d'une importance matérielle significative, fort de 1544 articles pour $88,4 \mathrm{ml}$, qui n' a fait l'objet d'aucune élimination.

Par ailleurs, ce fonds présente une cohérence dans sa composition formée de deux ensembles : une collection de carnets à souches des étalons nationaux et particuliers et des baudets de la période 1853 à 1960 (1 460 articles soit 85,3 ml), et des documents traitant autant de l'élevage chevalin que du fonctionnement du haras pour les années 1808 à 1999 (76 articles soit 3,1 ml). Cette dernière partie comprend des rapports adressés par le directeur aux autorités supérieures (ministre de l'Agriculture et préfet), les rapports de tournées très riches ${ }^{4}$ et les rapports sommaires rendant compte de la bonne marche du dépôt et des résultats obtenus, ainsi qu'un ensemble de correspondances et de documents concernant l'organisation intérieure du dépôt sur les plans comptable, matériel, immobilier et du personnel. Enfin, quelques pièces essentielles relatives au développement équin paraissent essentielles: les collections intégrales de registres matricules des étalons et de registres de la monte depuis la réouverture du dépôt, un registre sanitaire et des spécimens des cartes de saillies concernant à la fois les étalons du dépôt de Villeneuve et les juments de ce même dépôt saillies par des étalons des dépôts de Pau, Libourne, Pompadour, Rodez et Tarbes de la période 1891-1893.

Après cette phase archivistique, il nous fallait évaluer l'intérêt présenté par ces documents pour les chercheurs et étudier les informations contenues dans les carnets à souches de saillies. Pour ce faire, nous avons confronté ces typologies documentaires 
pour étudier la production de l'étalon "Souverain", pur sang du midi de passage au dépôt de Villeneuve-sur-Lot en 1911. C'est ainsi que chaque document apporte des informations spécifiques sur les points suivants :

- le registre matricule (fig. $\left.\mathbf{n}^{\circ} \mathbf{4}\right)$ suit l'étalon toute sa vie en précisant aussi sa production et son état de santé annuel,

\section{Figure 4}

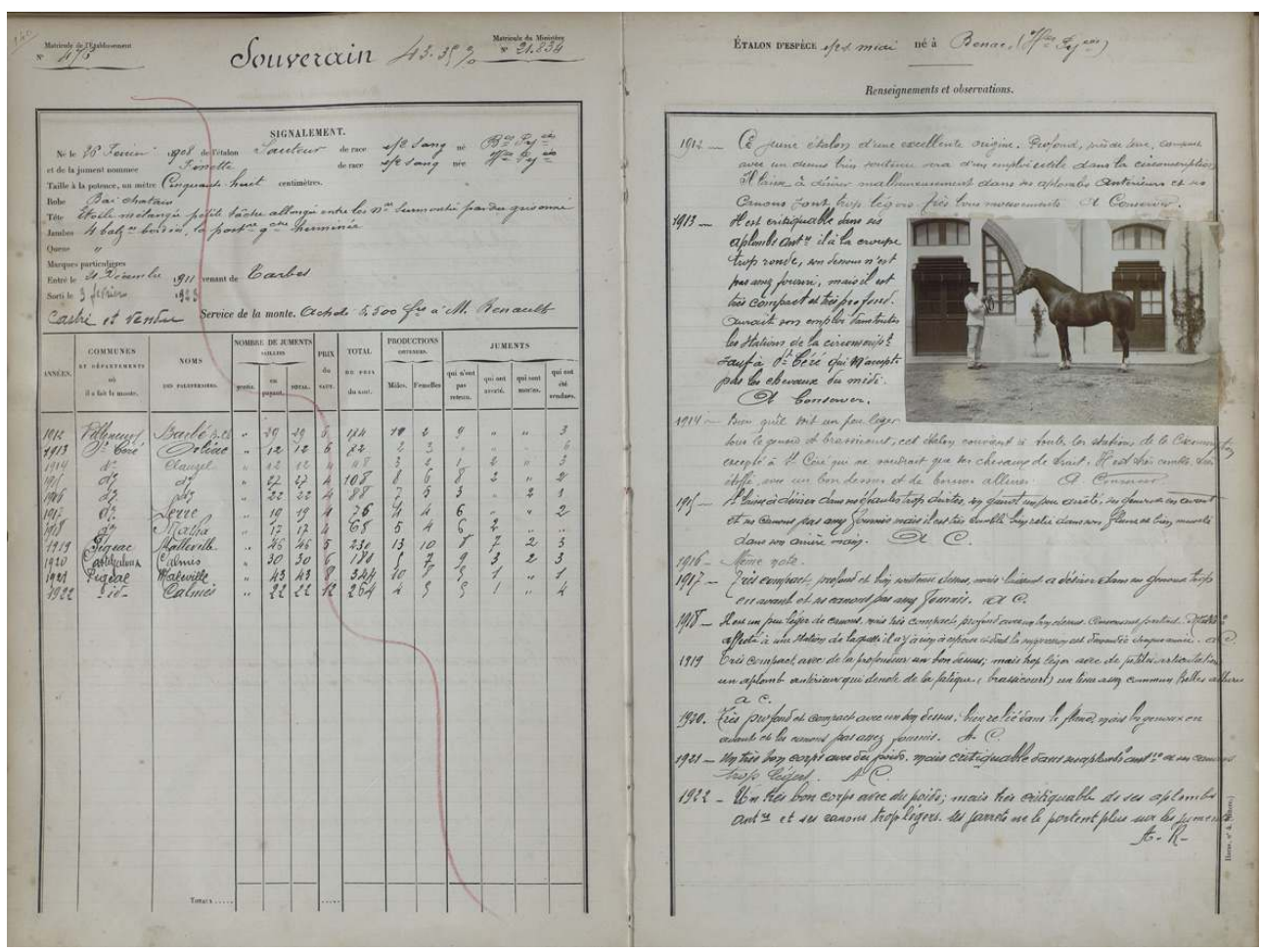

Dépôt d'étalons de Villeneuve-sur-Lot : feuillet matricule de l'étalon «Souverain », début XXe siècle. Document conservé aux Archives départementales de Lot-et-Garonne sous la cote 3 ETP 66.

Repro. Vigué, Marie. (c) Conseil général de Lot-et-Garonne, service des Archives départementales, 2011.

- le registre de la monte (fig. $\mathbf{n}^{\circ}$ 5) s'attache à une information plus statistique autour du prix du saut, du nombre de juments saillies (gratis et payant), du lieu de la monte, du nom du palefrenier, des productions obtenues, et des juments (qui n'ont pas retenu, qui ont avorté, qui sont mortes, qui ont été vendues), 


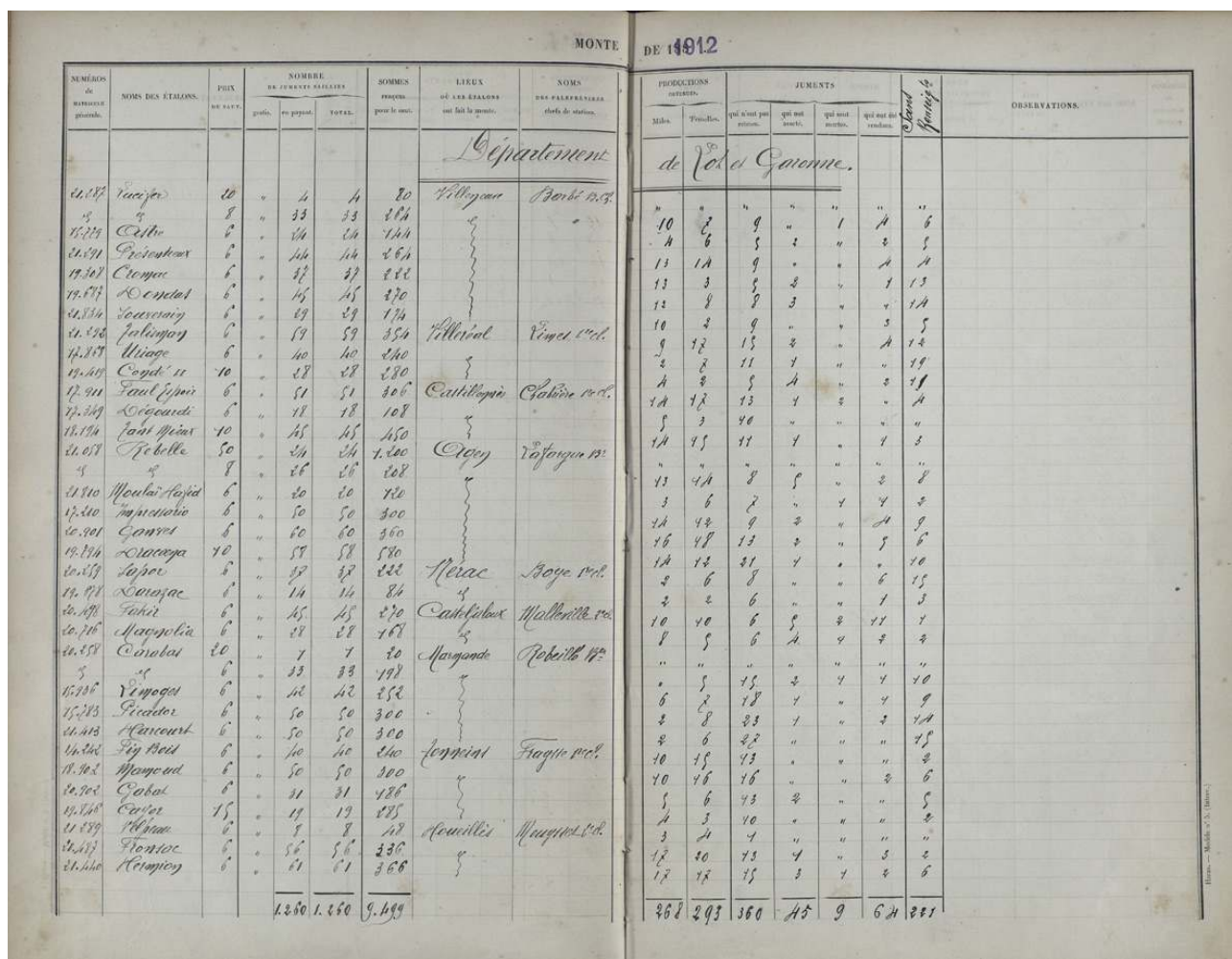

Dépôt d'étalons de Villeneuve-sur-Lot : feuille de la monte de l'année 1912. Document conservé aux Archives départementales de Lot-et-Garonne sous la cote 3 ETP 73.

Repro. Vigué, Marie. (C) Conseil général de Lot-et-Garonne, service des Archives départementales, 2011.

34 - la souche de saillie (fig. $\left.\mathbf{n}^{\circ} \mathbf{6}\right)$ renseigne seule sur la généalogie puisqu'elle précise le signalement de la jument, la date de la saillie, son propriétaire, la production née et la date de naissance du poulain. Ces cartes formaient, avec les souches, le carnet de saillies tenu par le dépôt. Le chef de station remettait ce certificat de saillie au propriétaire de la jument (comme quittance et déclaration de naissance sous couvert du maire de la commune) et conservait le talon. Le registre à talons était ensuite transmis au directeur du dépôt afin que les informations de la monte soient reportées dans le registre de la monte et le registre matricule. 
Figure 6

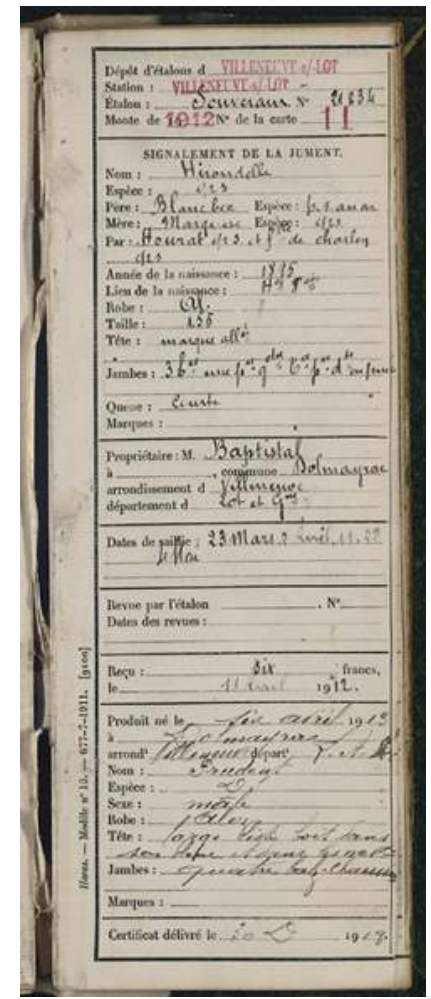

Dépôt d'étalons de Villeneuve-sur-Lot : souche de saillies de l'étalon «Souverain », 1912. Document conservé aux Archives départementales de Lot-et-Garonne sous la cote 3 ETP 846.

Repro. Vigué, Marie. (C) Conseil général de Lot-et-Garonne, service des Archives départementales, 2011.

Il paraît donc normal que le fonds du haras recèle la collection complète de ces documents. En y regardant de plus près, la carte et la souche (fig. $\mathbf{n}^{\circ} 7$ ) comportent strictement les mêmes informations. La souche de saillies servait donc à l'administration de preuve en tant que certificat de saillie et de déclaration de naissance, et de source pour actualiser les registres précités. D'ailleurs, l'administration attachait une importance toute particulière à leur conservation conformément aux dispositions réglementaires relatives au saut: "Les registres à talon de chaque année seront conservés avec soin " (fig. $\left.\mathbf{n}^{\circ} \mathbf{8}\right)$. La présence fortuite dans ce fonds de cartes de saillies pour les années 1891 à 1893 nous a donc permis de mesurer la valeur des souches, seul document que le dépôt de Villeneuve-sur-Lot se devait de posséder. 
Figure 7

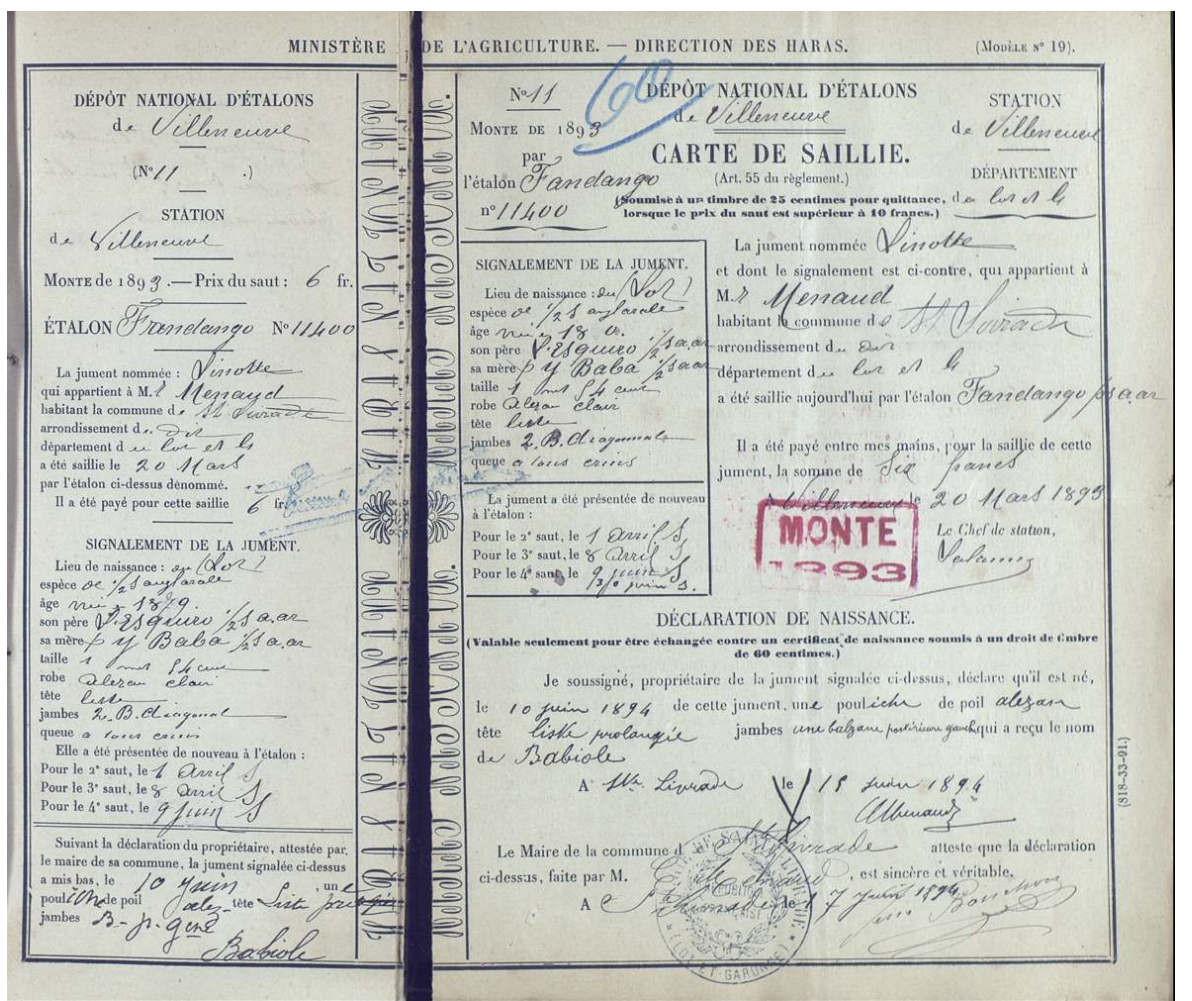

Dépôt d'étalons de Villeneuve-sur-Lot : souche et carte de la même saillie pratiquée par l'étalon "Fandango » sur la jument «Linotte», 1893. Documents conservés aux Archives départementales de Lot-et-Garonne sous les cotes 3 ETP 82 et 827.

Repro. Vigué, Marie. (c) Conseil général de Lot-et-Garonne, service des Archives départementales, 2011. 


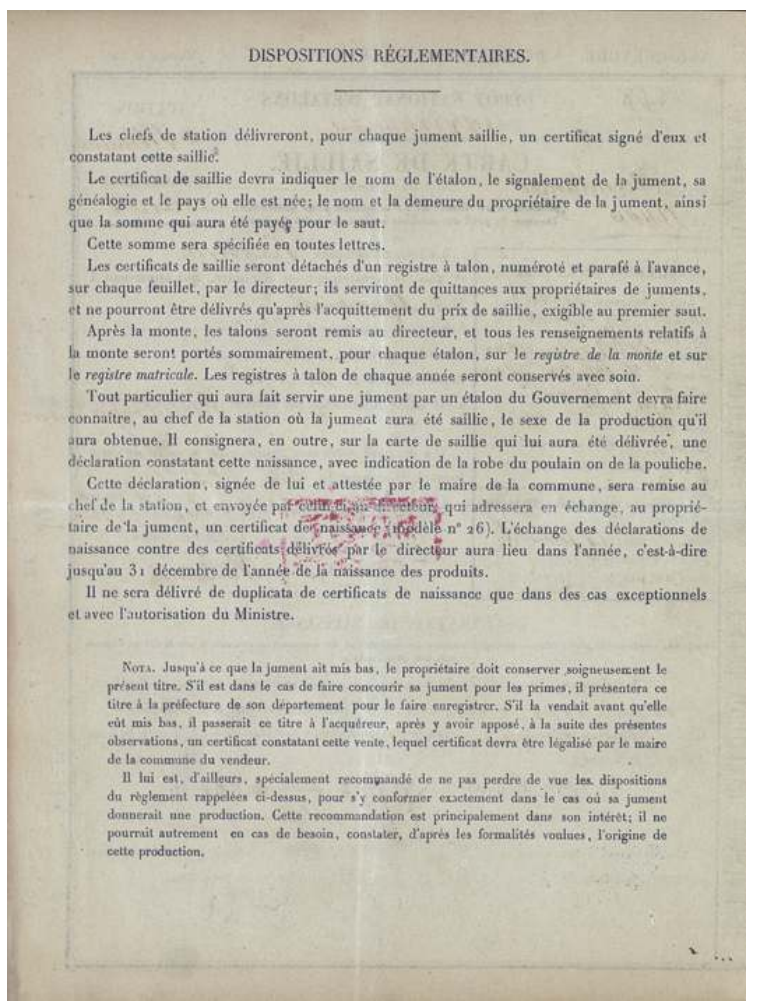

Dépôt d'étalons de Villeneuve-sur-Lot : carte de saillie (verso) de la jument « Linotte », 1893. Document conservé aux Archives départementales de Lot-et-Garonne sous la cote 3 ETP 82.

Repro. Vigué, Marie. (C) Conseil général de Lot-et-Garonne, service des Archives départementales, 2011.

$\mathrm{Au}$ final, les documents s'enrichissent mutuellement, rendant tout son sens et sa valeur historique à la collection de carnets à souches de saillies : l'ordre semble retrouvé dans la réunion de ces pièces créées à l'origine pour suivre avec précision les étalons et pourvoir à l'amélioration de la race des chevaux.

Dans un registre plus illustratif, signalons les nombreuses photographies noir et blanc du photographe villeneuvois de renom, Ray Delvert (fig. $\left.\mathbf{n}^{\circ} \mathbf{9}\right)\left(\mathbf{f i g} . \mathbf{n}^{\circ} \mathbf{1 0}\right)$, qui émaillent les registres matricules des étalons. 
Figure 9

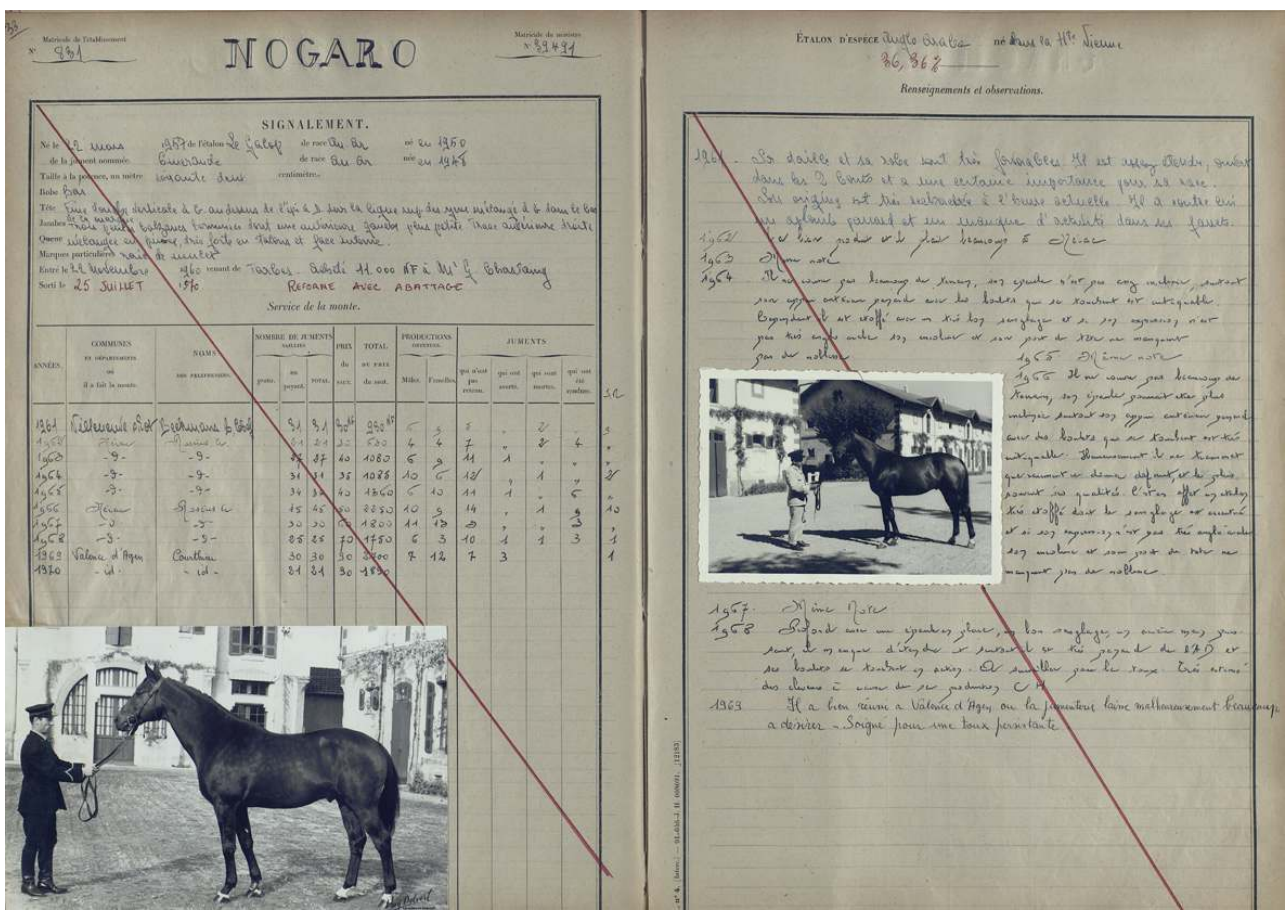

Dépôt d'étalons de Villeneuve-sur-Lot : photographie du feuillet matricule de l'étalon « Nogaro », milieu XXe siècle. Phot. Delvert, Ray. Document conservé aux Archives départementales de Lot-et-Garonne sous la cote 3 ETP 68

Repro. Vigué, Marie. (C) Conseil général de Lot-et-Garonne, service des Archives départementales, 2011. 


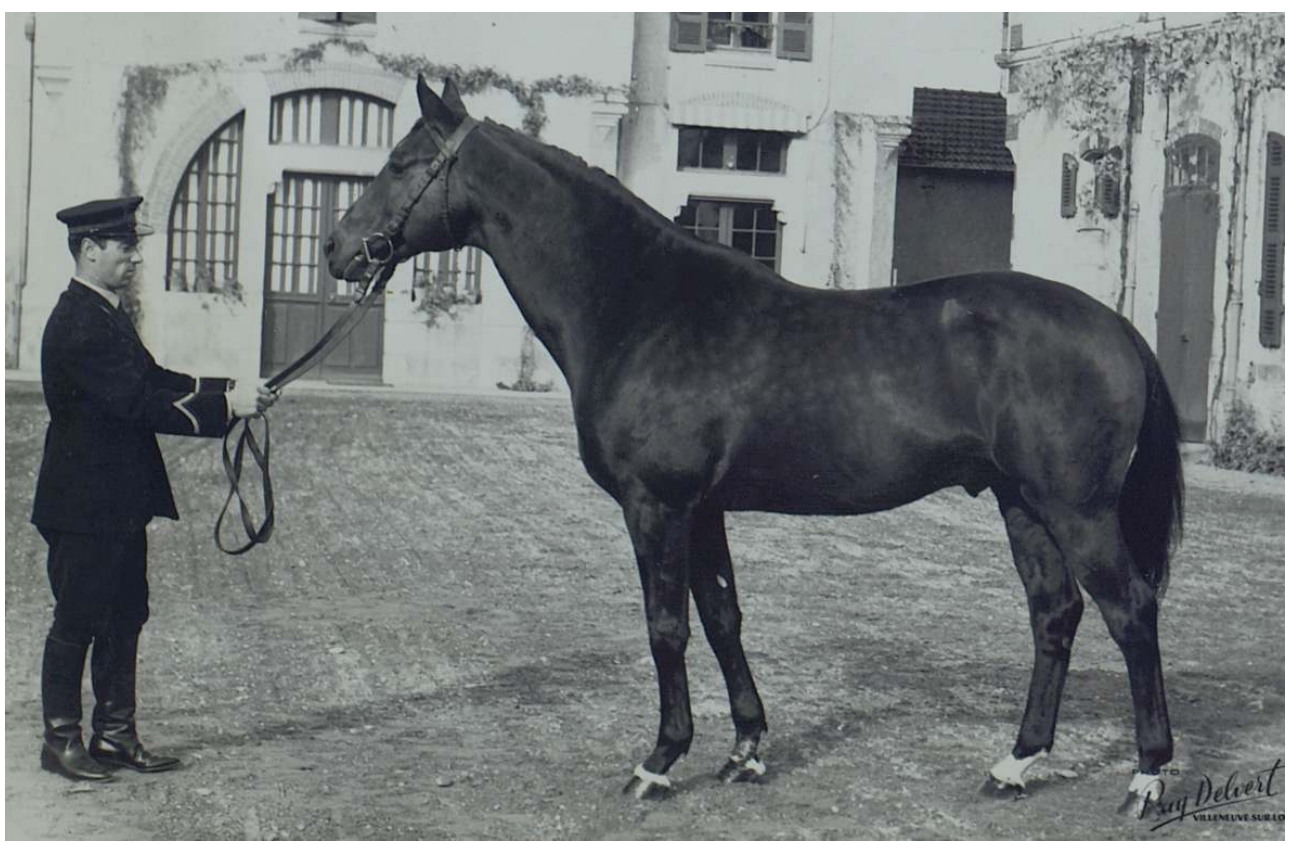

Dépôt d'étalons de Villeneuve-sur-Lot : agrandissement du cliché du feuillet matricule de l'étalon "Nogaro », milieu XXe siècle. Phot. Delvert, Ray. Document conservé aux Archives départementales de Lot-et-Garonne sous la cote 3 ETP 68.

Repro. Vigué, Marie. (c) Conseil général de Lot-et-Garonne, service des Archives départementales, 2011.

Portons enfin un regard plus critique sur le contenu du fonds, qui, comparé à d'autres dépôts, présente certaines lacunes préjudiciables à la connaissance de la première tranche d'activité au début du XIXe siècle. C'est ainsi que les documents relatifs au personnel sont particulièrement pauvres - aucune pièce sur les palefreniers et l'emploi du temps quotidien du personnel -, et qu'aucun document ne traite des concours, courses et sociétés hippiques.

\section{Après la reconstitution du fonds, quelles valorisation et perspectives?}

39 S'il est bien évidemment déplacé de parler d'un retour sur investissement, il faut reconnaître que ce fonds tarde à être exploité tant par le Haras que par les chercheurs. Signalons tout de même l'intérêt récent d'une étudiante bordelaise en architecture pour les bâtiments du dépôt.

Dans le cadre des relations que les Archives départementales entretiennent avec les services producteurs, notamment pour la gestion des archives intermédiaires, il serait souhaitable d'enrichir le fonds, en collectant les archives des années 1970-1990 encore conservées dans les murs du dépôt. Parallèlement, l'exhaustivité de l'inventaire gagnerait à intégrer les sources complémentaires relatives aux bâtiments des stations de monte, aux foires et courses hippiques des départements du Lot et de Tarn-et-Garonne. Les archives communales doivent contenir des informations sur les créations, fermetures et les réparations effectuées aux bâtiments des stations. 
41 Dans le cadre de ses activités, ce dépôt d'étalons pourrait utiliser ce patrimoine écrit pour renforcer les actions et manifestations à l'endroit du public. C'est ainsi que les stages de formation professionnelle aux métiers du cheval, s'adressant aux professionnels des centres équestres, et les stages de management à destination des entreprises pourraient puiser dans une approche historique. Dans le registre culturel, les manifestations en partenariat avec la ville (la Fête du cheval couplée aux Journées du patrimoine, «Les mardis des Haras » en lien avec l'office du tourisme, le salon du livre, le festival « Aux arts citoyens » en collaboration avec le théâtre Georges Leygues), tels les spectacles équestres organisés chaque été par une compagnie d'artistes en résidence, pourraient nécessiter un éclairage documentaire. Pour commencer, l'administration envisage de mettre en lumière son histoire dans un musée in situ intégrant des fac-similés de documents d'archives et des registres du Stud-book.

42 À l'appui des partenariats actuels où des classes de SEGPA ${ }^{5}$ et de l'EREA ${ }^{6}$ de Villeneuvesur-Lot ont participé à des ateliers d'écriture au Haras (dans lesquels le cheval est utilisé pour travailler le savoir-être des jeunes gens), les Archives départementales et le Haras nourrissent un projet d'action conjointe pour élaborer des parcours pédagogiques et des visites guidées pour le grand public. Ce dessein pourrait prendre place dans un projet commun initié par le lycée Bernard Palissy d'Agen, qui s'apprête à ouvrir, à la rentrée 2011, une classe Patrimoine. Dans le cadre d'un partenariat avec le Conseil général, les services des Archives départementales et du Patrimoine accompagneraient les élèves de cette classe pour les aider à réaliser des parcours muséographiques pour les classes de primaire et de collège en s'appuyant sur les patrimoines écrit, architectural et mobilier du Haras.

Afin d'aider ce dernier dans ses différents projets, les Archives départementales envisagent de numériser une partie de leurs fonds d'archives pour lui remettre une banque d'images.

\section{Conclusion}

Indubitablement, Guy Bideault a fait naître chez nous, archivistes, une prise de conscience sur l'intérêt de ces archives - affirmé en son temps par l'administration des Haras -, et nous a bien orientés dans le diagnostic des documents. Il s'agissait de saisir une opportunité (un chercheur s'intéressant à des documents, capable de les évaluer du fait de son métier et de ses besoins documentaires) tout en considérant le fonds dans son ensemble. Dès lors, les conditions étaient réunies et le temps était venu de s'y consacrer.

À l'aune de l'évolution des missions du haras, la préservation de la collection intégrale de carnets à souches de saillies paraît judicieuse et vitale pour comprendre l'activité principale de reproduction que cette institution a menée pendant deux cents ans. D'ailleurs, l'instruction du 16 janvier $2007^{7}$ a affirmé l'impérieuse nécessité de conserver toutes les souches des carnets de monte et de ne pas pratiquer l'échantillonnage car elles servent à établir la généalogie des animaux. Se priver de ces documents équivalait à se couper d'une information témoignant d'une mission fondatrice. Les Archives départementales ont donc joué la carte de la sécurité en tablant sur une conservation intégrale doublée de la collecte du reste des documents auprès du haras.

46 À présent, les documents sont ouverts à la communication: ils doivent servir aux chercheurs mais aussi au dépôt de Villeneuve-sur-Lot. Du reste, il est essentiel que ce 
dernier s'intéresse à son riche patrimoine et s'en empreigne pour ses actions culturelles, de communication et de formation.

En définitive, la coopération spontanée entre un chercheur, les Haras nationaux et les Archives de France a contribué à préserver et préparer l'exploitation historique de ce patrimoine écrit.

\section{NOTES}

1. - GUILLOTEL, Gérard. Les Haras nationaux. Paris : C. Lavauzelle, 1985, volume 3, p. 28.

2. - La collection des carnets à souches de saillies qui nous est parvenue ne commence qu'en 1853.

3. - <Lien vers http://www.cg47.org/archives/>.

4. - Ces documents s'articulent autour de quatre thématiques : la vie des stations, les créations, les suppressions, les besoins, le traitement des étalons ; les foires et concours de chevaux; les races de chevaux ; la distribution de primes destinées à l'encouragement de l'espèce chevaline.

5. - Sections d'enseignement général et professionnel adapté.

6. - Établissement régional d'enseignement adapté.

7. - Instruction $n^{\circ}$ DAF/DPACI/RES/2007/003 du 16 janvier 2007.

\section{RÉSUMÉS}

Comment l'intérêt de Guy Bideault, spécialiste de l'histoire du cheval, pour une collection «oubliée » de carnets à souches de saillies, versée il y a 26 ans de cela, a-t-il su convaincre les Archives départementales de Lot-et-Garonne de s'intéresser et de conserver ces documents sériels encombrants et inexploitables, pour dès lors s'attacher à reconstituer le fonds du dépôt d'étalons de Villeneuve-sur-Lot? L'étude s'attache non seulement aux phases d'évaluation archivistique des carnets de saillies, en lien avec les Haras nationaux et les Archives de France, et de collecte et traitement des archives du dépôt d'étalons, mais aussi à la cohérence du fonds avec les besoins documentaires des haras et des chercheurs.

How the interest of Guy Bideault, an expert in horse history, for a "forgotten" collection of noteb ooks of covering, registered 26 years ago, convinced the local archives of Lot-et-Garonne to focus and preserve these bulky and unusable serial documents, to attempt from then on to reconstitute the record group of the service station and stud of Villeneuve-sur-Lot? The study not only deals with the phases of archival appraisal of covering books, in connection with the Haras nationaux and France Archives, and of collecting policy and archival processing, but also with the consistency of the record group with the documentary needs of studfarm and researchers. 
INDEX

Mots-clés : Thesaurus des Archives de France, 2009, équidé, patrimoine écrit, reproduction animale, archivistique, cheval, haras

\section{AUTEUR}

PASCAL DE TOFFOLI

Archives départementales de Lot-et-Garonne, responsable des archives contemporaines padetoff@cg47.fr 